\title{
Document Thumbnails with Variable Text Scaling
}

\author{
A. Stoffel, H. Strobelt, O. Deussen and D. A. Keim \\ University of Konstanz, Germany
}

\begin{abstract}
Document reader applications usually offer an overview of the layout for each page as thumbnail views. Reading the text in these becomes impossible when the font size becomes very small. We improve the readability of these thumbnails using a distortion method, which retains a readable font size of interesting text while shrinking less interesting text further. In contrast to existing approaches, our method preserves the global layout of a page and is able to show context around important terms. We evaluate our technique and show application examples.
\end{abstract}

\section{Motivation}

The user interface of a typical document viewer application, such as Adobe Reader, consists of a detail view and one or more views for navigation within documents, such as a table of contents, and a thumbnail view providing page previews. In addition, most document viewer offer a keyword search functionality where the occurrence of keywords is highlighted in the detail view. However, the navigation views of document viewers (e.g. thumbnails) typically do not show the occurrence of keywords in the documents. So the user has to step through all occurrences of the keyword within the detail view by scrolling the pages.

To avoid this, we propose to highlight the keywords in the thumbnail view. This reduces the scrolling and the user is pointed directly to the interesting pages. In addition, thumbnails can be useful for retrieval tasks, if the users are trying to find something they already know [CvDRH99, DC02]. Due to the small size of text in thumbnails, the highlighting should in addition increase the size of the keywords and their context, at first to make the text better readable and second to allow a simple disambiguation of keywords by their context. For instance, it makes a difference if a text is about "user" or "user interface" but the keyword "user" would be highlighted in both.

The technique we present to create the thumbnails is a general distortion technique for document content that highlights words according to a user defined interest function. The global structure of a page, namely the position of images and columns, is preserved. An example is shown in Figure 1. In the keyword search application, an interest function is used that highlights the keywords and their context. Other applications might use a different interest function, for instance a sentiment score could be used to create thumbnails for sentiment analysis.

\section{Related Work}

Three different techniques are currently used for handling document overview and navigation: abstraction from the document with pixel based representations, thumbnails with different highlighting techniques, and semantic zooming.

A common pixel based technique is TileBars [Hea95], which visualizes the length of documents and the distribution of search terms within these documents with a rectangular pixel-based visualization. Byrd [Byr99] combines the scrollbar of the document view with a pixel visualization of search terms allowing the user to scroll directly to the occurrence of the terms. Both techniques do not show the context of the search terms and a user has to open the detail view in order to access this information.

Thumbnails, small version of the document or page, are commonly used for overview and navigation. The spacefilling thumbnail approach of Cockburn et al. [CGA06] avoids scrolling in the overview of a document, by positioning the thumbnails of all pages on a grid on the screen and resizing the thumbnails to fit the window size. Suh et al. [SWRG02] combined the thumbnails with popouts, which highlight search terms by rendering them in a readable size with a semi-transparently colored background above the original thumbnail. Woodruff et al. [WRM $\left.{ }^{*} 02\right]$ present an 


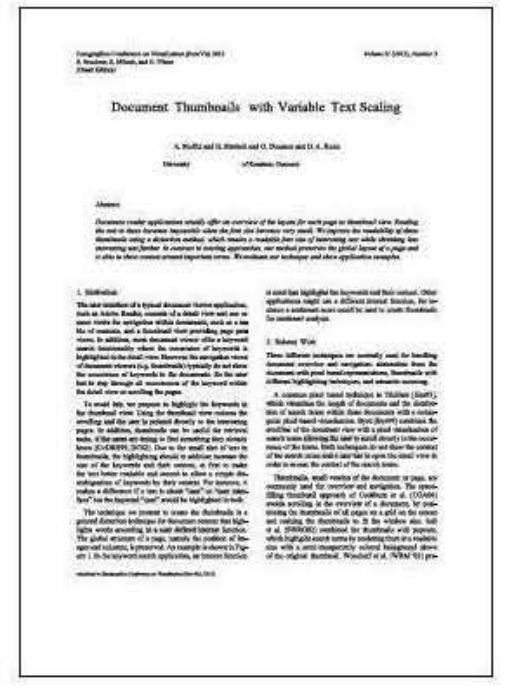

(a)

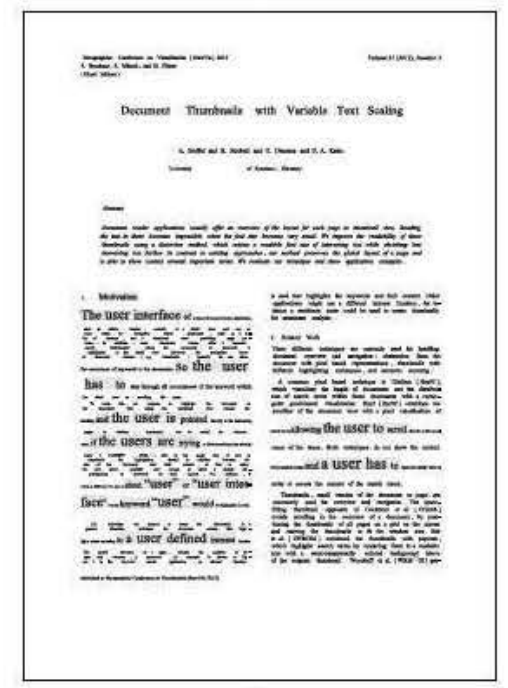

(b)

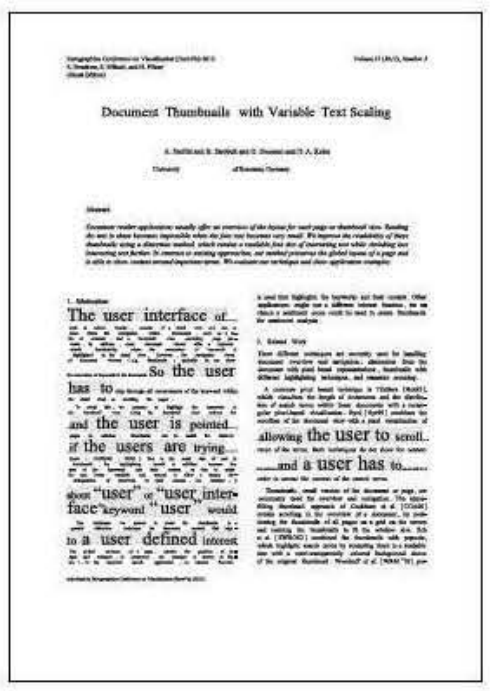

(c)

Figure 1: An example of a distorted thumbnail. a) The original thumbnail with highlights. b) The thumbnail generated with the zooming technique described in [BHDH95] c) The thumbnail generated with the proposed distortion technique.

enhanced thumbnail technique for web pages that, in addition to popouts for keywords, modifies the original HTML document to enlarge the size of headlines. However, highlighting with popouts leads to partial occlusion of the underlying document and the possibility of overlapping popouts in areas with many search terms.

The third technique, semantic zooming, has been used by several researchers. Several semantic zooming strategies for document thumbnails exist. Robertson and Mackinlay [RM93] place all pages of a document in a rectangular grid at thumbnail size. A user can magnify a single page using a fisheye lens for reading and checking context. Buchanan and Owen [BO08] increase the size of the important text such as headlines and recalculate the text layout while keeping the global page structure intact. Hornbæk and Frøkjær [HF01] use a fisheye technique for thumbnails, shrinking the uninteresting lines even further in order to highlight the interesting ones. Baudisch et al. [BLH04] combine a fisheye view in the vertical direction with popouts. The occlusions of the popouts are avoided by coloring the background of the interesting terms instead of painting the popouts above the document. Lam and Baudisch [LB05] use a thumbnail technique for overviews of web pages on small screen devices, where they remove common words or crop the text in the thumbnail in order to get a readable text in the final thumbnail. Instead of creating a thumbnail view and hiding information according to a semi-automatic decision, Baudisch et al. [BXWM04] use an interaction technique, which allows the user to define what information to show and what information to hide.
All the semantic zooming techniques change the document. In simple cases only the sizes of complete lines are changed; whereas more complex algorithms change the complete document according to the user's interest. Consequently, the page layout of the thumbnail changes and this could make it difficult for the user to relate positions in the thumbnail and the document view. The application of the fisheye algorithm in the vertical direction increases the height of the text, however as text is usually wider than high, the resizing is limited by the width of the view area.

Distortion algorithms developed for creating graph layouts are the most similar to our technique. Storey and Muller [SM96] distort the nodes in a graph according to a degree of interest; interesting nodes increase in size while the other nodes are shrunk. They present several distortion techniques that preserve the orthogonal or the proximity of nodes. Bartram et al. [BHDH95] use a similar technique for interactive zooming in hierarchical networks. On a drill down or a roll up in the hierarchy, the weights of the visible nodes are adjusted and the size is changed accordingly. Both techniques preserve the orthogonal ordering of the nodes and scale the node size according to an interest factor. But both techniques have problems when applied to text documents as the proximity of words are only relevant within a text line and not between text lines.

Our distorted page thumbnail approach combines plain page thumbnails variable text scaling and thereby avoids the occlusion and overplotting problems of popouts. In contrast to the previous fisheye approaches, a distorted thumbnail preserves the global page layout and allows a user to relate 
the positions in the thumbnail to the corresponding positions in the original page. The distortion is a derivative of the algorithm provided in [BHDH95] but is carefully designed to work best with textual data and preserve the page layout. Finally, the user has control over the amount of distortion.

\section{Design Considerations}

We now review the constraints associated with distorting the size of text.

D1 Overplotting and occlusion should be avoided and the overall page layout of the thumbnail should match the undistorted view, to aid navigation.

D2 The context of interesting text should be shown to give the user an additional hint of whether a passage in the document is of interest or not. A context in this case is typically a window of $n$ words before and after an interesting one.

D3 The most interesting text must be readable. In extreme conditions least interesting text may not be shown at all.

D4 The user should be able to control the size of the interesting text in the thumbnail.

In addition to the distortion, the background of interesting words can be colored to further increase their visibility. It is a common technique for text search functionalities in document views (e.g. Adobe Reader) to use a yellow background to highlight the matching words.

\section{Creating Distorted Page Thumbnails}

A distorted page thumbnail is created in a three step process. First, the degree of interest (DOI) for each word is calculated. The second step identifies the text columns forming the global page layout. In the last step the words of the segments are distorted and the final positions and size of the words are calculated.

\subsection{Overview of the Algorithm}

The input of the distortion algorithm is a document and an interest function that assigns a DOI to each word in the document. A simple interest function can be created from a string search by setting the DOI of a word to 1 if the word matches the search string, otherwise to 0. After applying the interest function to the document, the DOI along the text flow is smoothed by applying a Gaussian kernel. This smoothing distributes the degree of interest to the context of the interesting words and ensures the visibility of context in the final thumbnail. The user is able to control the size of interesting context by changing the size of the Gaussian kernel. The smoothing step is necessary to fulfill the requirements from D2, but might be omitted depending on the characteristics of the interest function.

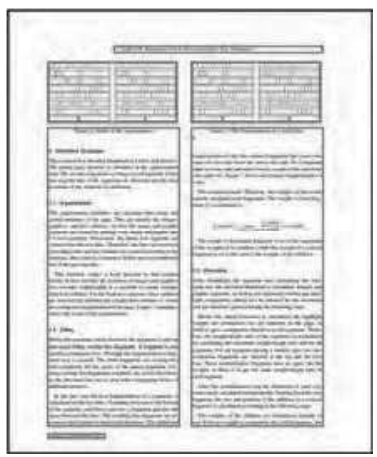

(a)

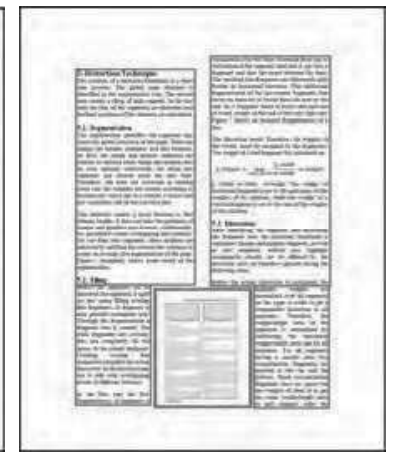

(b)
Figure 2: Results of the segmentation on different pages. The created segments are marked in red.

In the second step, the segments forming the global layout of the page are identified. These segments are the text columns, the images and the graphics on a page. For the distortion process, only the text columns are of interest, because images and graphics are not affected by the distortion. Figure 2 shows the result of the segmentation process for two different pages. The global page layout is preserved (D1) by distorting the content of the text columns and not their bounding boxes. The last step is to scale the size of words in a text column in the horizontal and vertical direction according to the assigned DOI.

\subsection{Distortion of Text}

The distortion algorithm is a modified version of the zooming algorithm for node networks described in Bartram et al. [BHDH95]. The original zooming algorithm works independently in horizontal and vertical directions by projecting the node boundaries on vertical and horizontal axes. The projected bounds are then moved along the axes such that the sizes of the intervals between them match the weight assigned to the corresponding nodes. An example for the horizontal intervals of a single line created by the projection is shown in Figure 3.

As text data is different to node networks, applying the zooming algorithm to the text column does not give an optimal result. For instance, the zooming algorithm preserves the horizontal order of nodes, which on text data is only required within a line and not within a complete text column. In order to improve the scaling of text data, we modify the zooming algorithm in the following way:

1. The vertical distortion is calculated on complete lines within a column instead of the single words. The horizontal scaling is then applied to the words on each line separately.

2. The user controllable parameter $\vartheta$ with $\vartheta \geq 1$ is intro- 


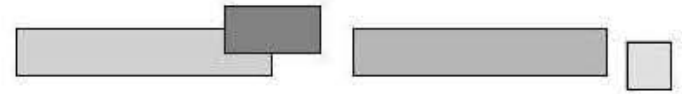

(a)

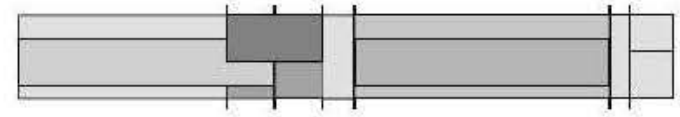

(b)

Figure 3: The horizontal intervals of a simple line. a) shows the bounding boxes of the words and b) shows the created intervals. The blue boxes are the interesting ones and the saturation corresponds to the degree of interest.

duced that limits the maximal width and height of a word to $w^{\prime} \leq \vartheta \cdot w$, respectively $h^{\prime} \leq \vartheta \cdot h$. Limiting the maximal size of a word has two effects: It introduces a variable that controls the distortion, which fulfills requirement D4. Setting this variable to 1 disables any distortion and the normal page thumbnails are generated. Secondly, limiting the size of the most interesting words leaves more space for the remaining words, especially for the context words, a requirement of D2.

3. The size of the most interesting words is increased at the expense of decreasing the size of the least interesting ones. We place the words in order from the highest to the lowest interest. This allows us to assign the optimal size to the high interest words. If too little space is available to show all words on a line, all the space is consumed by the high interest words and the low interest words are not shown at all. Figure 4 shows the results of the vertical, horizontal, and the combined distortion.

The distortion algorithm works as follows. Firstly, the algorithm determines the height of the lines in a text column. For this vertical distortion a DOI for each line in the text column must be calculated, because we do not use the words directly for adjusting the vertical size. In our case we define the DOI of a line $l_{i}$ as the maximal DOI of its words $t$ :

$$
l_{i} \text {.doi }=\max _{t_{j} \in l_{i}} t_{j} \text {. doi }
$$

The line bounds are then projected on the vertical axis. The intervals created by the projection belong either to a line or to a spacing. The DOI of a spacing interval is then set to the average DOI of its adjacent lines.

Next, we have to normalize the DOI in the whole document in order to get comparable line heights. The normalization is achieved by forcing the DOI to height ratio of the text columns in the document to the same value. We do not change the DOI of the lines, but add a DOI to spacing intervals so that after the normalization of the document $D$, all text columns $\{c \mid c \in D\}$ have the same DOI to height ratio $n_{v}$ :

$$
n_{v}=\max _{c_{k} \in D} \frac{c_{k} \cdot \text { doi }}{c_{k} \cdot \text { height }}
$$

The intervals are then distorted according to their DOI (see Algorithm 1). Starting from the interval with the maximal DOI, the height is adjusted linearly according to the total DOI and the available space. In contrast to the zooming algorithm of Bartram et al., the maximal height of an interval is limited to $\vartheta \cdot h$. If the new height $h^{\prime}$ of an interval would exceed the limit $\vartheta \cdot h$ with the linear scaling, the height is fixed to this limit and the DOIs of the remaining intervals are adjusted in order to preserve the normalized DOI to height ratio $n_{v}$ of the text column. Figure $4 \mathrm{~b}$ shows an example of vertical distortion.

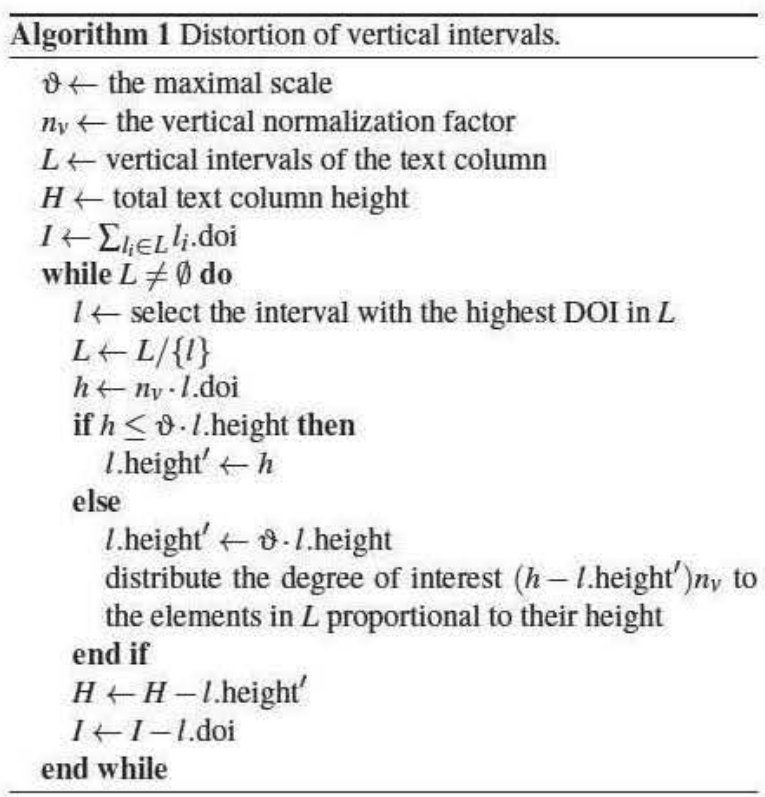

The horizontal distortion of words within a line is analogous to the vertical distortion of lines within a text column. Instead of projecting to the vertical axis, the word bounds are projected on the horizontal axis. For normalization, the DOIs of the space intervals are changed in order to get the same DOI to width ratio for all lines in the document. The final size of the intervals is then calculated with an adapted version of Algorithm 1. An example of the horizontal distortion is shown in Figure 4c.

Finally, the distorted page thumbnails are created from a combination of the vertical and horizontal distortions by scaling each word separately in a way that the word fits into the distorted vertical and horizontal borders and is aligned to the baseline of the text line. Figure $4 \mathrm{~d}$ shows the result after combining the vertical and horizontal distortions. 
For example, in a business letter processing scenario, appropriate metadata might include the recipient's name and address which could be used to route the letter to the recip-

(a) The original positions.

ha business letter processing scenario, propriate metadata might include the recipient's name and adderss which could be used to route the letter to the recip-

(c) Horizontal distortion only.
For example, in a business letter processing scenario, appropriate metadata might include the recipient's name and address which could be used to route the letter to the recip(b) Vertical distortion only.

\section{a business letter processing scenario, propriate metadata might include the recipient's name and route the letter to the}

(d) Combined horizontal and vertical distortion.

Figure 4: Example of the distortion of three lines. The saturation of the blue color shows the degree of interest of the different boxes.

\section{Evaluation}

Our distortion technique for generating document thumbnails has the advantage that important words are prominent and overplotting or occlusion problems are avoided. This advantage comes at the cost of a size constraint for interesting terms. While the size of popouts can be independent of the page and text column size, the size of a distorted term is not. This raises the question how small a thumbnail can get while the interesting terms and their contexts are still readable. Another question we investigate is how our technique differs from the original zooming algorithm of Bartram et al. [BHDH95].

The size of a word is directly related to its readability. If the algorithm scales up a word by a factor $\alpha$, the page can be shrunk by the same factor $\alpha$ and the word has the same size as before. In order to evaluate the distorted thumbnail algorithm, we compare the size of the words before and after the distortion. For this comparison we calculate the area factor $A=\frac{w^{\prime} \cdot h^{\prime}}{w \cdot h}$ that expresses how much a word was scaled. As a final measure, we use the median of the area factor of the interesting words and of the context words in a document. The median is chosen rather than the arithmetic mean, because it guarantees that half of the words have an area factor greater or equal to this value.

For a fair comparison of our distortion algorithm with the original zooming algorithm of Bartram et al., the zooming algorithm is not applied to a whole page, but to each text column separately. Additionally, the size of a text column is kept fixed and not changed by the algorithm. These two modifications ensure that the zooming algorithm does not change the layout or dimensions of the page, as out distortion algorithm does.

The amount of distortion depends mainly on the available space and the distribution of interesting terms. The available space is determined by the page format and layout of a page. For instance, the possible amount of horizontal distortion is larger with a single column layout than on a page with a two columns. In order to test the influence of the page layout on the distortion we use two document collections: the MICAI 2011 proceedings with a single column and the EuroVis 2011 proceedings with a two column layout. The EuroVis 2011 proceedings contain 538 pages with 48047 lines (average 10.57 words per line). The MICAI 2011 proceedings contain 591 pages with 23300 lines (average 13.12 words per line). In order to emulate different distribution of interesting terms, we decided not to search for single terms, but to use randomly generated interest functions with different distribution of interesting terms. After filtering stop words, we equally distribute the DOI over the remaining words with different densities and vary the kernel size.

\subsection{Results and Discussion}

Figure 5 shows the area factor on different term distributions at a kernel size of 5 . The parameter $\vartheta$ of the distortion is set to $\vartheta=2$. For the zooming technique of Bartram et al. the weight of an interesting word is set to 3.75 times the weight of a normal word. These parameters are adjusted in a way that the median area factor is closest to 4.0 when $0.1 \%$ of terms are of interest. The results in Figure 6 show the area factor over different kernel sizes with $2 \%$ of interesting terms. The parameters of the distortion and of the zooming algorithm are the same as before.

Figure 5 shows the difference between the zooming and the distortion algorithm according to the density of interesting words. In the case of Figure 5a, the area factors of interesting words behave similar for both techniques: with a few interesting words the area factor is high and degrades as the number of interesting words increases. The area factor becomes 1 when either the height or the width of the words cannot be increased, because both algorithms preserve the aspect ratio. The difference between the two algorithms is 


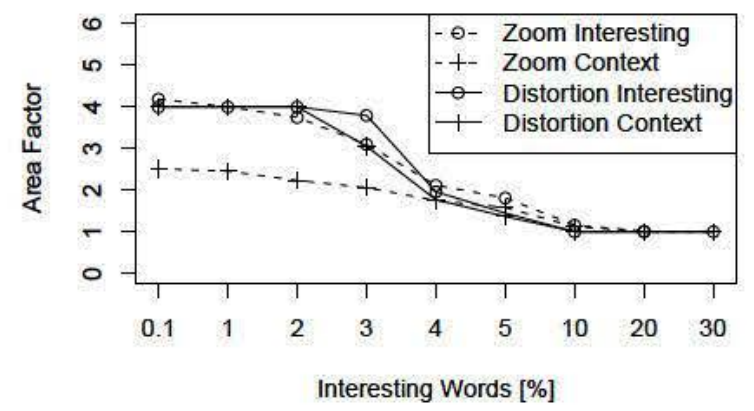

(a) EuroVis 2011

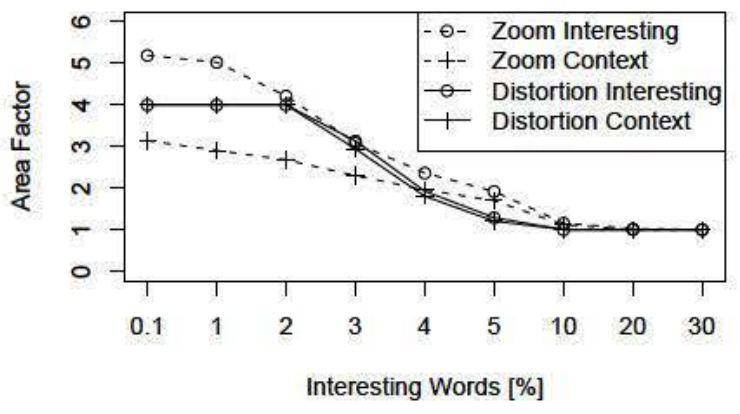

(b) MICAI 2011

Figure 5: The area factor over different distribution of interesting terms.

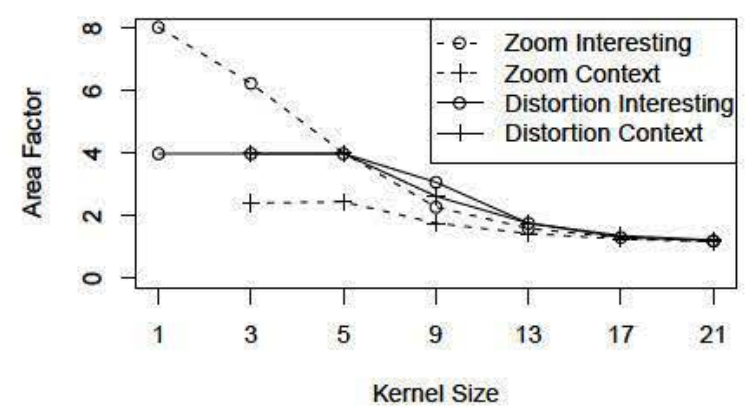

(a) EuroVis 2011

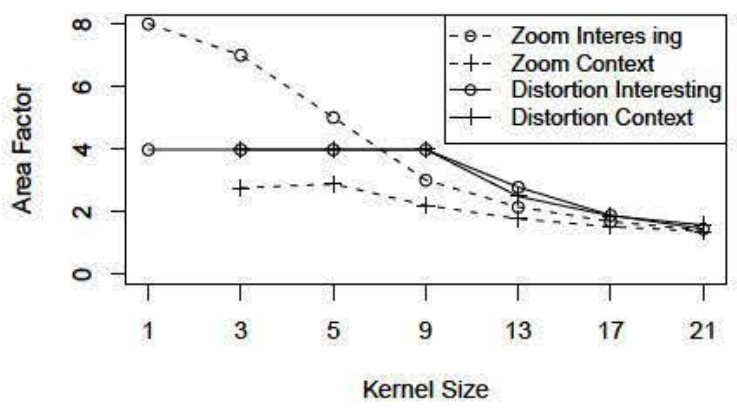

(b) MICAI 2011

Figure 6: The area factor for different kernel sizes.

the size of the context words when the number of interesting words is low. In this case the distortion is able to assign more space to the context words than the zooming algorithm. In the case of $0.1 \%$ interesting words, the size of the interesting words has reached the maximal allowed value and thus the context words can use the additional space. This effect becomes clearer in the results of the single column layout in Figure $5 \mathrm{~b}$. The distortion is able to increase the size of the context words with the same factor as the interesting words for up to $2 \%$ of interesting words. The zooming algorithm on the other hand does not have an upper bound for the size of words. This leads to the situation that the most interesting words consume the majority of available space and less space is available for the context words.

The results in Figure 6 compare the two techniques for different kernel sizes. For large kernel sizes, the two algorithms produce similar results on both collections. But for small kernel sizes, the results of the algorithms are different. As before, the distortion assigns more space to the context words, because of the upper bound of word size.
As expected, the area factor for the single column layout of the MICAI proceedings is slightly larger than for the two column layout of the EuroVis proceedings, because the width of a line limits the amount of horizontal distortion. As a single column layout allows wider lines, more distortion can be applied to the text of the MICAI proceedings.

The comparison of our distortion algorithm with the original zooming algorithm shows that for high densities of interesting terms both algorithms behave similar. In this case too little words are of low interest and neither the zooming nor the distortion algorithm is able to increase the size of interesting words. In case, the interesting terms are sparsely distributed, both algorithms increase the size of the words differently. The zooming algorithm linearly scales the words according to the assigned interest, which results in larger interesting words and smaller context words. In contrast, our distortion algorithm limits the maximal size of the interesting words and is able to increase the size of the context words with the same factor. As a result, the context words 
are larger and therefore better readable with our distortion algorithm then with the zooming algorithm.

\section{Application Examples}

\subsection{Keyword Search in Documents}

Searching for keywords in documents is a common task for uses of document reader applications. With the distorted document thumbnails this task is supported by highlighting the search terms and their context in the thumbnails. The required interest function simply maps the search terms to 1 and all the other terms to 0 . The context of the search terms is also interesting, so this interest function is smoothed with a Gaussian kernel that also highlights the context words of each occurrence of the search terms in the document.

An example of a keyword search result is shown in Figure 1. In this case we are looking for the keyword "user" on the first page of this paper. The thumbnails show the result for different techniques. In Figure 1a the thumbnail is created by scaling the page to the desired size and highlighting the keywords with a yellow background. Through this highlighting the user can identify the positions on the page where the keywords occur. But the small size of the thumbnails makes the text barely readable.

Using the zooming algorithm, this improves (see Figure 1b) with an increase in the size of the keywords and their context and a corresponding decreasing the size of the other text. Consequently, it is easier to spot the keywords and even the context is readable due to the effect of smoothing the DOI with the Gaussian kernel. The keyword "user" gets the maximal size and the size of the context words decrease with additional distance from the keyword.

Figure 1c shows the distorted page thumbnail. While the distortion maintains a normalization of the keyword size, the size relation between keywords and context words does not reflect the difference in interest. This is the advantage of the distortion, because it is able to assign similar size to both the context and the keywords.

The advantage of showing the context is obvious when comparing the different thumbnails in Figure 1. Through the context shown in Figure 1b and Figure 1c the usage of the word "user" becomes clear. The first occurrence refers to a user interface and not to the user itself. Other occurrences show what the user can do with page thumbnails. For instance, the user can jump, find and navigate within a document.

\subsection{Document Overview}

In Figure 7 an overview of a EuroVis 2011 paper [vdZLBI11] was created by highlighting the terms of the title. The resulting thumbnails give a user, in addition to the page layout, a hint about the content of the different pages. For instance, the introduction on the first page starts with talking about molecular visualizations. The related work section on the second page discusses first visualization techniques and then structural abstractions, and a reader interested in continuity would want to read page four. This is a clear benefit of the distortion thumbnails, because on a normal page thumbnail the text would not be readable at all.

Figure 7 also shows the limitation of the distorted thumbnails. The maximal possible size of a word is restricted by the size of the text column. But in order to get readable words, the size of the thumbnails cannot go smaller than a certain value. The second parameter influencing the amount of distortion is the distribution of the interesting words in the documents. In the best case, the interesting words are equally distributed with many uninteresting words in-between, which can be shrunk. In the worst case, every word is of interest and hence no distortion is allowed at all and results in a normal scaled thumbnail with yellow highlights.

In this example both problematic cases exists. Firstly, the text columns with limited space are the page headers, which only contain a single line and the text cannot increase in size vertically. Secondly, areas with many interesting words can be found, e.g. the left column on the third or seventh page. However, in this example the thumbnail size is less than a sixteenth part of the original page size and a lot of interesting text is still readable. This is achieved by relaxing the normalization of the word size. Instead of normalizing the size in the whole document, we normalized the average size of interesting words and allowed local variation when not enough space is available.

\section{Conclusion}

The presented document thumbnails with variable text scaling are able to highlight words and their context in thumbnails. The algorithm avoids overplotting and occlusions by distorting the page content according to a user defined interest function. The distortion preserves the basic functionality of a thumbnail and shows the global layout of the page, allowing a user to skip over uninteresting pages and to find a page by its layout. In contrast to existing approaches, the distorted thumbnails are able to show keywords together with their context. This is achieved by adapting an existing scaling algorithm for node networks to the text domain and focusing on readable size for the most interesting text.

For future research we would like to investigate the question of which contexts make sense for keyword searches. In this paper we defined a context for search term as the adjacent terms with the effect that also stop words are highlighted that have no meaning, e.g. determiners. A different definition of context could exclude stop words or use only nouns. In addition, a more intelligent solution to areas with many interesting words should be considered. In the current 
1172
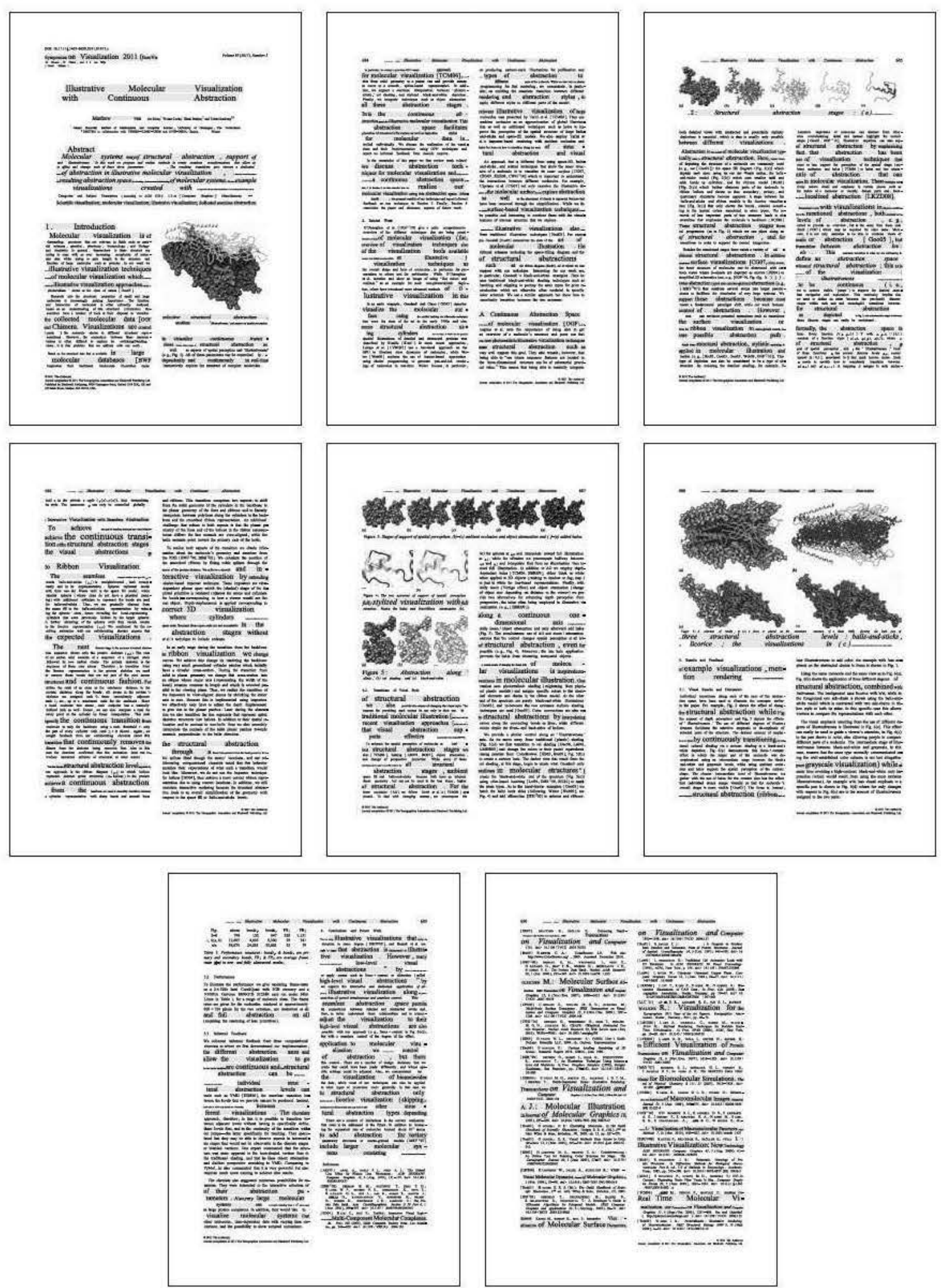

Figure 7: Distorted page thumbnails created for one of the EuroVis2011 papers [vdZLBI11]. The thumbnails show the distribution of the title terms in the document. 
algorithm we fit the interesting words in the page layout, which limits the maximal size of the words. One possibility would be to allow the algorithm to introduce line breaks. In a user study, a different representation of very small words and lines should also be considered. In the current implementation the text is simply scaled to a very small size, a different representation could instead indicate small lines and words by gray rectangles.

\section{References}

[BHDH95] Bartram L., Ho A., Dill J., Henigman F.: The Continuous Zoom: a constrained fisheye technique for viewing and navigating large information spaces. In Proceedings of the 8th annual ACM symposium on User interface and software technology (1995), UIST '95, ACM, pp. 207-215. 2, 3, 5

[BLH04] Baudisch P., LeE B., Hanna L.: Fishnet, a fisheye web browser with search term popouts: a comparative evaluation with overview and linear view. In Proceedings of the working conference on Advanced visual interfaces (New York, NY, USA, 2004), AVI '04, ACM, pp. 133-140. 2

[BO08] BuChANAN G., OwEN T.: Improving Skim Reading for Document Triage. In Proceedings of the second international symposium on Information interaction in context (New York, NY, USA, 2008), IIiX '08, ACM, pp. 83-88. 2

[BXWM04] BAUdisch P., XIE X., WANG C., MA W.-Y.: Collapse-to-Zoom: Viewing Web Pages on Small Screen Devices by Interactively Removing Irrelevant Content. In Proceedings of the 17th annual ACM symposium on User interface software and technology (New York, NY, USA, 2004), UIST '04, ACM, pp. 91-94. 2

[Byr99] BYRD D.: A Scrollbar-based Visualization for Document Navigation. In Proceedings of the fourth ACM conference on Digital libraries (New York, NY, USA, 1999), DL '99, ACM, pp. 122-129. 1

[CGA06] Cockburn A., Gutwin C., Alexander J.: Faster Document Navigation with Space-Filling Thumbnails. In Proceedings of the SIGCHI conference on Human Factors in computing systems (New York, NY, USA, 2006), CHI '06, ACM, pp. $1-10.1$

[CvDRH99] CZERWinski M., VAn DANTZICH M., RobertSON G., HofFman H.: The Contribution of Thumbnail Image, Mouse-over Text and Spatial Location Memory to Web Page Retrieval in 3D. In Proceedings of the INTERACT'99 conference (1999). 1

[DC02] DZIADOsz S., CHANDRASEKAR R.: Do thumbnail previews help users make better relevance decisions about web search results? In Proceedings of the 25th annual international ACM SIGIR conference on Research and development in information retrieval (New York, NY, USA, 2002), SIGIR '02, ACM, pp. 365-366. 1

[Hea95] HeARst M. A.: TileBars: Visualization of Term Distribution Information in Full Text Information Access. In Proceedings of the SIGCHI conference on Human factors in computing systems (New York, NY, USA, 1995), CHI '95, ACM Press/Addison-Wesley Publishing Co., pp. 59-66. 1

[HF01] HornbæK K., FrøKJÆR E.: Reading of Electronic Documents: The Usability of Linear, Fisheye, and Overview+Detail Interfaces. In Proceedings of the SIGCHI conference on Human factors in computing systems (New York, NY, USA, 2001), CHI '01, ACM, pp. 293-300. 2
[LB05] LAM H., BAUdisch P.: Summary Thumbnails: Readable Overviews for Small Screen Web Browsers. In Proceedings of the SIGCHI conference on Human factors in computing systems (New York, NY, USA, 2005), CHI '05, ACM, pp. 681-690. 2

[RM93] Robertson G. G., Mackinlay J. D.: The Document Lens. In Proceedings of the 6th annual ACM symposium on User interface software and technology (New York, NY, USA, 1993), UIST '93, ACM, pp. 101-108. 2

[SM96] Storey M. D., Müller H. A.: Graph Layout Adjustment Strategies. In Proceedings of the Symposium on Graph Drawing (1996), GD '95, Springer-Verlag, pp. 487-499. ACM ID: 728600.2

[SWRG02] SuH B., WoodrufF A., Rosenholtz R., Glass A.: Popout Prism: Adding Perceptual Principles to Overview+Detail Document Interfaces. In Proceedings of the SIGCHI conference on Human factors in computing systems: Changing our world, changing ourselves (New York, NY, USA, 2002), CHI '02, ACM, pp. 251-258. 1

[vdZLBI11] VAN DER ZWAN M., LUEKS W., BEKKER H., ISENBERG T.: Illustrative Molecular Visualization with Continuous Abstraction. Computer Graphics Forum 30, 3 (2011), 683-690. 7, 8

[WRM*02] WOOdrufF A., Rosenholtz R., MORrison J. B., Faulring A., Pirolli P.: A Comparison of the Use of Text Summaries, Plain Thumbnails, and Enhanced Thumbnails for Web Search Tasks. J. Am. Soc. Inf. Sci. Technol. 53 (January 2002), 172-185. 1 